\title{
Research progress regarding the role of long non-coding RNAs in osteosarcoma (Review)
}

\author{
YANLI ZHANG ${ }^{*}$, YANCHUAN PU*, JIN WANG, ZICAI LI and HULIN WANG \\ Department of Orthopedics, Wuwei People's Hospital, Wuwei, Gansu 733000, P.R. China
}

Received February 11, 2020; Accepted May 29, 2020

DOI: $10.3892 / \mathrm{ol} .2020 .11807$

\begin{abstract}
Osteosarcoma is a malignant tumor that occurs in children and adolescents. Although treatments for osteosarcoma have improved, the likelihood of survival remains low for most patients with metastasis and recurrence. Elucidating the mechanism underlying the development of osteosarcoma and chemotherapy resistance will be important to improve diagnosis and treatment. Long non-coding RNAs (lncRNAs), which are longer than 200 nucleotides in length and do not encode for proteins, have been shown to play a regulatory role in the occurrence and development of osteosarcoma, and are expected to serve as biomarkers and molecular targets. This review discusses the progress in the study of the role of lncRNAs in osteosarcoma, and highlights the recent developments in this field.
\end{abstract}

\section{Contents}

1. Introduction

2. Classification and biological function of lncRNAs

3. Promotive role of lncRNAs in the occurrence and development of OS

4. Inhibitory effect of lncRNAs on the development of OS

5. The role of IncRNAs in OS resistance

6. Summary and prospects

\section{Introduction}

Osteosarcoma (OS) is a malignant tumor that accounted for $15 \%$ of all diagnosed malignancies in children and adolescents

Correspondence to: Professor Yanchuan $\mathrm{Pu}$, Department of Orthopedics, Wuwei People's Hospital, 24 Xuanwu Street, Liangzhou, Wuwei, Gansu 733000, P.R. China

E-mail: puych918@163.com

*Contributed equally

Key words: osteosarcoma, long non-coding RNA, microRNA, chemotherapy resistance, biomarkers in 2015 that seriously affects the health of these groups of individuals worldwide (1). Epidemiological statistics show that the survival rate of patients with primary OS is only $65-70 \%$ and the survival rate after metastasis is only $19-30 \%$; thus, current clinical treatments do not effectively cure OS $(2,3)$. Currently, $>90 \%$ of patients with OS are treated with limb-preserving surgeries (3). However, the high rates of recurrence, metastasis, and mortality of spinal OS result in an extremely low 5-year survival rate (3). Therefore, identifying novel biomarkers involved in the pathogenesis of OS is of great significance.

Long non-coding RNAs (lncRNAs) are functionally defined as gene transcripts more than 200 nucleotides in length that have no protein-encoding potential. IncRNAs act as major regulatory RNAs, that regulate gene expression at the epigenetic or genetic level. In particular, lncRNAs have been found to play an important role in the occurrence, development and angiogenesis of tumors (4). With the development of next-generation sequencing technology, the OS-associated mutation spectrum has been deciphered, including the expression profiles of lncRNAs. A large number of lncRNAs have been found to play an important role in the occurrence, development and drug resistance in OS $(5,6)$. Further studies on the role of lncRNAs in OS will improve the understanding and ability to treat this disease. This review summarizes current knowledge regarding the function of IncRNAs at the molecular level and the implications for OS research and therapy.

\section{Classification and biological function of IncRNAs}

lncRNAs are classified as sense lncRNAs, antisense lncRNAs, bidirectional lncRNAs, intron lncRNAs, intergenic lncRNAs, or enhancer lncRNAs, according to the positional association between lncRNAs and protein-coding genes in the genome (7).

At present, the regulation of genes by lncRNAs mainly occurs at the transcriptional and post-transcriptional levels (8). lncRNAs can regulate gene expression through chromatin modification, remodeling, histone modification and nuclear body localization (9). In cooperation with the SWI/SNF complex, IncRNAs can also change the structure of chromosomes, thus directly affecting gene expression (10). lncRNAs can directly induce DNA methylation and damage, which can also affect gene expression $(11,12)$. Finally, lncRNAs can regulate gene expression through complex interactions with microRNAs (miRNAs) (13) (Fig. 1). 
3. Promotive role of IncRNAs in the occurrence and development of $\mathrm{OS}$

The occurrence and development of OS is a complex process involving many factors. While the molecular mechanisms of these physiological and biological processes have not been fully elucidated, it is clear that IncRNAs play an important role $(5,6,14)$.

IncRNAs directly promote the occurrence and development of $O S$. Dong et al (15) revealed that the upregulation of bladder cancer associated transcript 1 (BLACAT1) predicted an unfavorable prognosis for patients with OS. The downregulation of BLACAT1 inhibited cell proliferation and invasion, whereas the upregulation of BLACAT1 accelerated cell proliferation and invasion. More importantly, BLACAT1 accelerated the proliferation and migration of OS cells by regulating the phosphorylation of STAT3. Zhao et al (16) showed that HLA complex P5 (HCP5) acts as an oncogene in OS, and the upregulation of HCP5 promoted cell proliferation and invasion, the epithelial-mesenchymal transition (EMT), and the development of OS. Chen et al (17) showed that LOXL1 antisense RNA 1 (AS1) predicted the clinical progression and poor prognosis in patients with OS, and functions as an oncogenic lncRNA, regulating cell proliferation, cell cycle, migration, and invasion via the PI3K/AKT pathway. Su et al (18), revealed that ELK1-induced upregulation of MIR100HG predicted poor prognosis and promoted the progression of OS by epigenetically silencing large tumor suppressor kinase (LATS)1 and LATS2, and inactivating the Hippo pathway. Another study suggested that the lncRNA hepatocellular carcinoma upregulated lncRNA could promote the occurrence of bone tumors by enhancing the proliferation, invasion, migration and expression of EMT-associated factors of OS (19). Meanwhile, nuclear paraspeckle assembly transcript 1 was shown to be significantly upregulated in OS tissue, indicating that it could effectively promote the proliferation and metastasis of OS cells (20). Meanwhile, Gu et al (21) confirmed that HOXD-AS1 could interact with enhancer of zeste homolog 2 (EZH2), thereby decreasing the expression of p57 and exacerbating OS. Yang et al (22), found that upregulated forkhead box P4-AS1 promoted OS proliferation, migration and the cell cycle, but inhibited apoptosis; this effect was achieved through the interaction with lysine-specific demethylase 1 and EZH2, and further downregulation of LATS1. Yu et al (23), demonstrated that antisense non-coding RNA in the INK4 locus (ANRIL) affects OS cell proliferation, invasion and apoptosis by regulating the AKT pathway. Ye et al (24), found that nicotinamide nucleotide transhydrogenase (NNT)-AS1 could inhibit the proliferation, migration and invasion of OS cells, as well as tumor growth, by inhibiting the expression of NNT-AS1. Zhang et al (25), also demonstrated that prostate cancer-associated transcript 1 could promote cell proliferation, invasion and migration, as well as the EMT, in OS. Li et al (26), showed that colorectal neoplasia differentially expressed (CRNDE) plays an oncogenic role in OS cell lines, and CRNDE can act as a carcinogen by regulating Notch1 signaling and the EMT in OS. Overall, lncRNAs mainly promote the occurrence and development of OS by regulating the proliferation, migration, invasion and EMT of OS cells.

The interaction between lncRNAs and miRNAs promotes the occurrence and development of OS. miRNAs, which have a maximum length of 200 nucleotides, are the most well-known and well-studied non-coding RNAs. Since these molecules appear to play an important role in cellular biology, further investigations are necessary to elucidate their true functions (27).

Jiang et al (28), found that the expression of differentiation antagonizing non-protein coding RNA was increased in OS cells, mediated cancer resistance, enhanced AXL invasion and metastasis by competitively binding with miRNA-33a-5p, and targeting AXL degradation by miRNA-33a-5p via the PI3K/AKT pathway. Wang et al (29), also demonstrated that DANCR could induce the expression of miRNA-335-5p and miRNA-1972, thereby promoting ROCK1-mediated proliferation and transfer through competing endogenous RNA (ceRNA) networks, and thus the development of OS. Ma et al (30), found that UCA1 could act in the ceRNA network of miRNA-582 to positively regulate the expression of CREB1, thereby enhancing the EMT through the PI3K/AKT/mTOR pathway mediated by CREB1, and promoting invasion and metastasis of OS cells. Meanwhile, Liao et al (31) demonstrated that small nucleolar RNA host gene 16 might negatively regulate the expression of miRNA-98-5p. This regulation could effectively reverse the inhibitory effects of miRNA-98-5p on the proliferation, migration, invasion, cell cycle and apoptosis of OS cells. Another report showed that distal-less homeobox 6-AS1 could inhibit the proliferation and metastasis of OS cells by targeting the miRNA-641/HOXA9 signaling pathway and acting as a ceRNA (32). Sun et al (33), found that MALAT1 could regulate the proliferation and metastasis of OS cells by competitively binding with miRNA-34a/c-5p and miRNA-449a/b. Deng et al (34), showed that lncRNA-small nucleolar RNA host gene 1 (SNHG1) plays an oncogenic role in OS. By activating ROCK1, the PI3K/AKT pathway and the EMT, IncRNA-SNHG1 can downregulate the expression of miRNA-101-3p and promote the proliferation, migration and invasion of cancer cells. Wang et al (35), also confirmed that SNHG1, acting as an endogenous sponge, inhibits the activity of miRNA-326 by increasing the expression of its target gene Nob1. Dai et al (36), confirmed that OIP5-AS1 acts as a miRNA-223 sponge to trigger CDK14 expression, thus promoting OS tumorigenesis. Li et al (37) ,demonstrated that TUG1 could accelerate the proliferation, migration, and invasion of OS cells by competitive diffusion of miRNA-219a-5p, leading to the upregulation of PIK3CA and activation of AKT signaling. Another study showed that TUG1, as a ceRNA of miRNA-132-3p, promoted SOX4 expression and inhibited the expression of miRNA-132-3p, leading to increased proliferation and decreased apoptosis of OS cells (38). Moreover, Wang et al (39) found that HOXA-AS2 can promote OS proliferation, migration, invasion and EMT transformation by regulating the expression of miRNA-520c-3p. Deng et al (40), demonstrated that SNHG7 inhibits the growth of tumor suppressor 


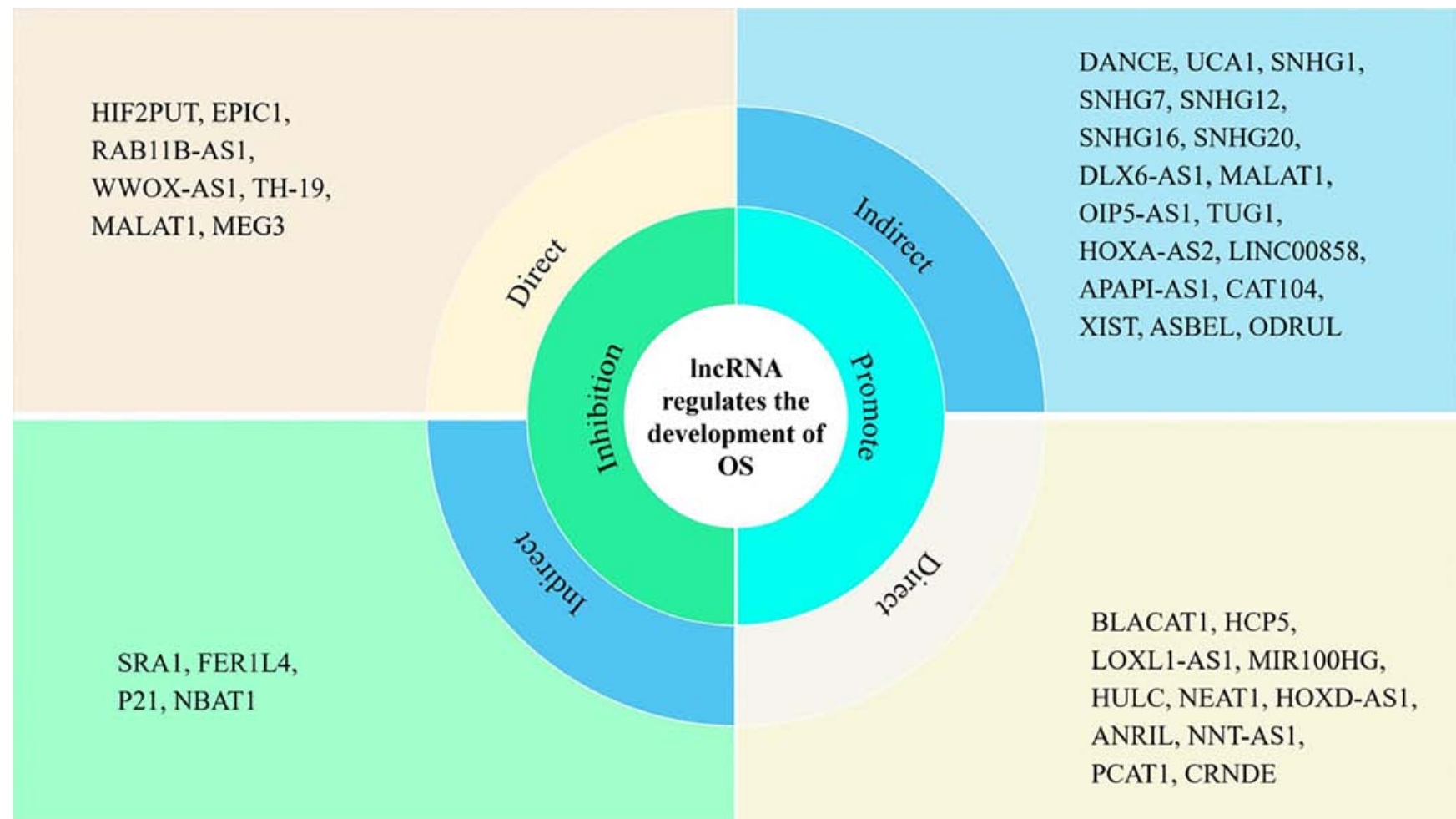

Figure 1. Different lncRNAs play a role in promoting or inhibiting the development of OS, directly or indirectly. Systematic classification of lncRNAs with different effects could provide a basis for application of lncRNAs in the diagnosis and treatment of OS. OS, osteosarcoma; lncRNAs, long non-coding RNAs.

miRNA-34a and upregulates its target gene, which could induce cell proliferation, cell cycle arrest and apoptosis. $\mathrm{Gu}$ et al (41), revealed that LINC00858 regulates the OS phenotype by acting as a ceRNA of miRNA-139 to enhance CDK14 expression. Li et al (42), found that actin filament-associated protein 1-AS1 promoted the proliferation and invasion of OS cells by inhibiting miRNA-4695-5p and activating Tcf4- $\beta$-catenin signaling. Xia et al (43) confirmed that inhibition of CAT104 could significantly inhibit the proliferation, migration and invasion of OS cells by regulating the expression of miR-381 and the downstream ZEB1, JNK, and Wnt/ $\beta$-catenin pathways. Yang et al (44) showed that $\mathrm{X}$ inactive specific transcript (XIST) was upregulated in OS tissues and cells. XIST affected the proliferation, invasion and EMT of OS cells by regulating miRNA-195-5p/Yes-associated protein signaling. Zhao et al (45) found that ASBEL could induce $\mathrm{PI} 3 \mathrm{~K} / \mathrm{AKT} / \mathrm{GSK} 3 \beta$ and MEK/ERK signaling, as well as the activation, proliferation, migration, and invasion of OS cells by inhibiting the expression of miRNA-21. Zhou et al (46) found that SNHG12 regulated Notch expression by acting as a molecular sponge of miRNA-195-5p, thereby activating Notch signaling in OS and playing a regulatory role in the occurrence and progression of OS. Zhu et al (47) showed that the IncRNA osteosarcoma doxorubicin-resistance related up-regulated IncRNA could effectively absorb miRNA-3182 by upregulating MMP2. This regulation directly led to the loss of the inhibitory effects of miRNA-3182 on OS proliferation, migration, invasion and tumor growth, and indirectly promoted the progression of OS. The experimental data of another study showed that the IncRNA SNHG20/miRNA-139/RUNX family transcription factor 2 (RUNX2) axis promoted the occurrence and apoptosis of OS cells through the mitochondrial apoptotic pathway (48). Overall, lncRNAs and miRNAs act as crucial regulators in the occurrence and development of OS by acting as a ceRNA.

\section{Inhibitory effect of IncRNAs on the development of OS}

Although most members of the IncRNA family promote the occurrence and development of OS, a number of lncRNAs have been shown to actively inhibit the development of cancer. After systematically collating and analyzing the results described in this review, a number of IncRNAs have been found to have significant inhibitory effects on the occurrence and development of OS.

IncRNAs directly inhibit the occurrence and development of OS. Zhao et al (49) showed that the lncRNA HIF2PUT could inhibit the proliferation and migration of OS cells, and the self-renewal of OS stem cells, by upregulating HIF-2 $\alpha$. Zhao et al (50) showed that upregulation of EPIC1 could promote ubiquitin-mediated degradation of myocyte-specific enhancer factor 2, an oncogene important in the development of OS. Therefore, EPIC1 can effectively inhibit the occurrence and progression of OS. Chen et al (51) showed that RAB11B-AS1 expression was negatively correlated with RAB11B expression, and thus, RAB11B-AS1 could significantly inhibit the occurrence and development of OS. The results of $\mathrm{Qu}$ et al (52) showed that IncRNA WW domain containing oxidoreductase (WWOX)-AS1 inhibited the proliferation, migration and invasion of OS cells. The overall survival rate of the high WWOX-AS1 group was higher compared with 
Table I. Expression and association of lncRNAs in osteosarcoma.

\begin{tabular}{lllccc}
\hline Chemotherapeutic drug & IncRNA & Deregulation & Genes/proteins affected & Signaling pathway & (Refs.) \\
\hline Gemcitabine & PVT1 & Up & microRNA-152 & c-MET/PI3K/AKT & $(61)$ \\
& KCNQ1OT1 & Up & DNMT1/Kcnq1 & - & $(62)$ \\
CDDP & TUG1 & Up & - & MET/Akt & $(63)$ \\
& OIP5-AS1 & Up & miR-340-5p/LPAAT $\beta$ & PI3K/AKT/mTOR & $(64)$ \\
& ANRIL & Not analyzed & miR-125a-5p & STAT3 & $(65)$ \\
DXR & HOTTIP & Up & - & Wnt/ $/$-catenin & $(69)$ \\
methotrexate & SNHG12 & Up & miR-320a/MCL1 & - & $(66)$ \\
& FOXC2-AS1 & Up & FOXC2/ABCB1 & - & $(67)$ \\
& LUCAT1 & Up & miR-200c/ABCB1 & - & $(68)$
\end{tabular}

Drug resistance of osteosarcoma is one of the most important problems in the clinical treatment of osteosarcoma. Different lncRNAs play an important role in the chemoresistance of osteosarcoma, which is expected to be a breakthrough point to solve this problem. lncRNA, long non-coding RNA; miR, microRNA.

the low WWOX-AS1 group. Therefore, WWOX-AS1 may be associated with the occurrence and development of OS. Furthermore, the molecular mechanism underlying the inhibition of OS by WWOX-AS1 is associated with genes activated by RUNX2. Moreover, Zhao and Ma (53) found that TH19 expression affected the migration and invasion of OS cells by activating the NF- $\mathrm{KB}$ pathway. Zhang et al (54), showed that metastasis associated lung adenocarcinoma transcript 1 (MALAT1) inhibited the occurrence and progression of OS by enhancing the expression of angiogenic factors, such as vascular endothelial growth factor A and fibroblast growth factor 2, via positive regulation of the MALAT1/mTOR/hypoxia-inducible factor-1 $\alpha$ pathway. Zhang et al (55) and Sun et al (56) found that MEG3 could inhibit the growth and metastasis of OS cells by inhibiting the Notch and TGF- $\beta$ signaling pathways. Overall, IncRNAs directly inhibit the occurrence and development of OS by regulating the proliferation, angiogenesis, and metastasis of OS cells.

The interaction between IncRNAs and miRNAs inhibits the occurrence and development of OS. Guo et al (57) found that the lncRNA steroid receptor RNA activator 1 plays an anti-tumor role in OS, via the sponge miRNA-208a, since it decreases cell migration, invasion and proliferation, and promotes apoptosis. Fei et al (58) demonstrated that fer-1 like family member 4 inhibits the proliferation, colony formation, migration and invasion of OS cells by regulating the miRNA-18a-5p/PTEN pathway. Han et al (59) showed that lncRNA-p21 decreased the proliferation of OS cells in vivo and in vitro, by inhibiting the expression of miRNA-130b, thus enhancing the anti-tumor effects of PTEN/pAKT. Yang et al (60) showed that the expression of IncRNA-neuroblastoma associated transcript 1 (NBAT1) is downregulated in OS tissues and cell lines. The downregulated expression of NBAT1 in OS is closely associated with the clinical stage, lymph node metastasis, and prognosis. In addition, NBAT1 was shown to inhibit OS tumor growth and metastasis in vivo by binding miRNA-21. Overall, IncRNAs and miRNAs may inhibit the occurrence and development of OS, by acting as a ceRNA.

\section{The role of IncRNAs in OS resistance}

At present, the treatment of OS is primarily based on chemotherapy following surgical excision of lesions. In recent decades, with the overall improvement in human health and the development of clinical imaging technology, lesions in some patients with OS are detected early and surgery can be performed before metastasis occurs. However, most patients still require chemotherapy, which contributes to the rapid development of resistance. Chemotherapy resistance of OS is the primary reason for treatment failure at present; determining the intrinsic mechanism of resistance has become an important avenue of research. In addition to their role in the occurrence and development of OS, IncRNAs have also been shown to mediate OS resistance (Table I).

Sun et al (61), demonstrated that the IncRNA plasmacytoma variant translocation 1 (PVT1) could enhance the resistance of OS to gemcitabine by directly targeting and downregulating activation of the c-MET/PI3K/AKT pathway by miRNA- 152 . These results demonstrated that PVT1 not only promotes the occurrence and development of OS, but also enhances the resistance of patients to conventional chemotherapy drugs. Qi et al (62), found that knockdown of the lncRNA KCNQ1OT1 inhibited the proliferation, migration and invasion of OS cells, and upregulated potassium voltage-gated channel subfamily Q member 1 through DNA methyltransferase 1, to promote apoptosis and chemosensitivity to cisplatin (DDP). Therefore, KCNQOT1 may be an important promoter of the occurrence, development and chemotherapy resistance of OS. Zhou et al (63), demonstrated that TUG1 also promoted DDP resistance and inhibited DDP-induced cytotoxicity and apoptosis of Saos-2/DDP and MG-63/DDP cells. Song et al (64), showed that opa interacting protein 5 (OIP5)-AS1 silencing led to the accumulation of miR-340-5p and thus inhibited mRNA translation, decreased the protein expression of lysophosphatidic acid acyltransferase $\beta$ (LPAAT $\beta$ ), and inactivated the PI3K/AKT/mTOR signaling, resulting in decreased cisplatin resistance in OS. In conclusion, OIP5-AS1 induced LPAAT $\beta /$ PI3K/AKT/mTOR signaling by mutagenizing miRNA-340-5p, resulting in cisplatin resistance in OS. Li and 
Zhu (65), demonstrated that ANRIL mediated the proliferation, apoptosis and cisplatin resistance of OS cells by targeting miRNA-125a-5p/STAT3. Zhou et al (66), demonstrated that SNHG12 could enhance OS resistance to doxorubicin (DXR) by inhibiting the expression of miRNA-320a and promoting the expression of MCL1. Knockdown of MCL1 could enhance the sensitivity of OS cells to DXR. In addition, the upregulation of MCL1 could attenuate the sensitivity to DXR induced by the knockdown of mimic RNA-320a and SNHG12. Zhang et al (67), showed that forkhead box C2 (FOXC2)-AS1, and in particular its FOXC2-overlapping region, exerted these functions by forming RNA-RNA double strands with FOXC2, further increasing the RNA and protein levels of FOXC2 and promoting the expression of ATP binding cassette subfamily B member 1 (ABCB1), a classical multi-drug resistance gene, ultimately resulting in OS resistance to doxorubicin. Han and Shi (68), revealed that lung cancer associated transcript 1 enhances OS resistance to methotrexate by modulating the miRNA-200c ABCB1 pathway. A study by Li et al (69), showed that HOXA distal transcript antisense RNA, which is overexpressed in OS, plays an important role in OS proliferation, accelerating cell cycle progression and inducing chemoresistance via the Wnt $/ \beta$-catenin pathway. Therefore, the drug resistance of OS is one of the most important problems in the clinical treatment of OS. Different lncRNAs play an important role in the chemoresistance of OS, which are expected to be a breakthrough point to solve this problem.

Resveratrol is a natural antitoxin that has previously been reported to have selective anti-tumor effects on a variety of tumor cells, including OS. Hu et al (70), showed that taurine up-regulated 1 (TUG1) was abnormally upregulated in adriamycin-resistant OS cells. Resveratrol can inhibit the proliferation, and promote the apoptosis, of drug-resistant OS cells by inhibiting AKT signaling mediated by TUG1.

\section{Summary and prospects}

As described in this review, there is ample evidence to support the view that lncRNAs play an important role in the occurrence, growth, invasion, metastasis and drug resistance of OS. Furthermore, it is apparent that different members of the IncRNA family play different roles in OS. Therefore, at present, lncRNAs cannot be defined as cancer initiators or anticancer agents in OS. This will require an understanding of the common mechanisms of lncRNA regulation through exploring the single regulatory roles of different lncRNAs in detail, and determining their associations and mechanism of internal feedback regulation. In this way, important laboratory results can be translated into clinically feasible treatments.

However, it is also important to recognize that current research on the role of IncRNAs in OS is still at the exploratory stage, and there is insufficient evidence to support its use for clinical applications. Nevertheless, it is believed that the current experimental results support lncRNAs as future biomarkers and therapeutic targets in the diagnosis and prognosis of OS.

lncRNAs are used as biomarkers for early diagnosis of $O S$. IncRNAs play an important regulatory role in OS tumorigenesis, tumor cell proliferation, invasion, migration, apoptosis and angiogenesis. IncRNAs can be secreted and circulated in bodily fluids. As lncRNAs secreted in the circulation are resistant to RNAse, PCR could be used to clinically detect the presence of IncRNAs. Recent reports have indicated IncRNAs as good candidates for tumor markers with high specificity, sensitivity and non-invasive characteristics $(71,72)$.

It is believed that the detection of IncRNAs using readily available clinical specimens, such as blood and articular puncture fluid, could revolutionize the early diagnosis of OS. If early diagnosis of OS can be made, the efficacy of early clinical treatments will be improved.

IncRNAs serve as a molecular target for OS biotherapy. Cancer treatment based on biological targeting technology has been gradually accepted and promoted by clinicians. This type of precise cancer treatment, which shows good therapeutic effects and fewer side effects, represents an important breakthrough. IncRNAs can be targeted using small interfering RNA, antisense oligonucleotides, ribozymes, aptamers and miRNAs; these methods have long been used to target important cancer-associated genes, and are at different stages of clinical trials $(73,74)$. Overall, lncRNAs, which regulate the development of OS, may be a molecular target for OS biotherapies.

\section{Acknowledgements}

Not applicable.

\section{Funding}

This study was financially supported by the Chinese Medicine Administration Research Project of Gansu province (grant no. GZK-2019-46).

\section{Availability of data and materials}

Not applicable.

\section{Authors' contributions}

YZ and YP conceived the review. YZ, ZL, and JW wrote the review. ZL and $\mathrm{HW}$ revised the review. YP proofread the manuscript and revised the manuscript for important intellectual content. All authors have read and approved the final manuscript.

\section{Ethics approval and consent to participate}

Not applicable.

\section{Patient consent for publication}

Not applicable.

\section{Competing interests}

The authors declare that they have no competing interests. 


\section{References}

1. Mirabello L, Troisi RJ and Savage SA: Osteosarcoma incidence and survival rates from 1973 to 2004: Data from the surveillance, epidemiology, and end results program. Cancer 115: 1531-1543, 2009.

2. Meyers PA, Heller G, Healey JH, Huvos A, Applewhite A, Sun $M$ and LaQuaglia M: Osteogenic sarcoma with clinically detectable metastasis at initial presentation. J Clin Oncol 11: 449-453, 1993

3. Kager L, Zoubek A, Pötschger U, Kastner U, Flege S, Kempf-Bielack B, Branscheid D, Kotz R, Salzer-Kuntschik M, Winkelmann W, et al: Primary metastatic osteosarcoma: Presentation and outcome of patients treated on neoadjuvant Cooperative Osteosarcoma Study Group protocols. J Clin Oncol 21: 2011-2018, 2003.

4. Peng $\mathrm{Z}$, Liu $\mathrm{C}$ and $\mathrm{Wu} \mathrm{M}$ : New insights into long noncoding RNAs and their roles in glioma. Mol Cancer 17: 61,2018.

5. Luo Z, Xiao L, Li J, Dong B and Wang C: Integrative analysis reveals driver long non-coding RNAs in osteosarcoma. Medicine (Baltimore) 98: e14302, 2019.

6. Ren K, Ni Y, Li X, Wang C, Chang Q, Li Y, Gao Z, Wu S, Shi X, Song J, et al: Expression profiling of long noncoding RNAs associated with vasculogenic mimicry in osteosarcoma. J Cell Biochem 120: 12473-12488, 2019.

7. Li Y, Egranov SD, Yang L and Lin C: Molecular mechanisms of long noncoding RNAs-mediated cancer metastasis. Genes Chromosomes Cancer 58: 200-207, 2019.

8. Akhade VS, Pal D and Kanduri C: Long noncoding RNA: Genome organization and mechanism of action. Adv Exp Med Biol 1008: 47-74, 2017.

9. Deans C and Maggert KA: What do you mean, 'epigenetic'? Genetics 199: 887-896, 2015

10. Chen Z, Gao Y, Yao L, Liu Y, Huang L, Yan Z, Zhao W, Zhu P and Weng H: LncFZD6 initiates Wnt/ $\beta$-catenin and liver TIC self-renewal through BRG1-mediated FZD6 transcriptional activation. Oncogene 37: 3098-3112, 2018.

11. Deng J, Mueller M, Geng T, Shen Y, Liu Y, Hou P, Mamillapalli R, Taylor HS, Paidas M and Huang Y: Correction: H19 LncRNA alters methylation and expression of $\mathrm{Hnf} 4 \alpha$ in the liver of metformin-exposed fetuses. Cell Death Dis 10: 592, 2019.

12. Jain AK, Xi Y, McCarthy R, Allton K, Akdemir KC, Patel LR, Aronow B, Lin C, Li W, Yang L and Barton MC: LncPRESS1 Is a p53-regulated LncRNA that safeguards pluripotency by disrupting SIRT6-mediated De-acetylation of histone H3K56 Mol Cell 64: 967-981, 2016.

13. Tam C, Wong JH, Tsui SKW, Zuo T, Chan TF and Ng TB: LncRNAs with miRNAs in regulation of gastric, liver, and colorectal cancers: Updates in recent years. Appl Microbiol Biotechnol 103: 4649-4677, 2019.

14. Léveillé N and Baglio SR: Exosome-transferred LncRNAs at the core of cancer bone lesions. Crit Rev Oncol Hematol 139: 125-127, 2019

15. Dong $\mathrm{Z}$ and Wang Y: LncRNA BLACAT1 accelerates the proliferation and migration of osteosarcoma cells through regulating STAT3. Pathol Res Pract 215: 571-579, 2019.

16. Zhao W and Li L: SP1-induced upregulation of long non-coding RNA HCP5 promotes the development of osteosarcoma. Pathol Res Pract 215: 439-445, 2019.

17. Chen S, Li W and Guo A: LOXL1-AS1 predicts poor prognosis and promotes cell proliferation, migration, and invasion in osteosarcoma. Biosci Rep 39: BSR20190447, 2019.

18. Su X, Teng J, Jin G, Li J, Zhao Z, Cao X, Guo Y, Guo M, Li X, Wu J, et al: ELK1-induced upregulation of long non-coding RNA MIR100HG predicts poor prognosis and promotes the progression of osteosarcoma by epigenetically silencing LATS1 and LATS2. Biomed Pharmacother 109: 788-797, 2019.

19. Zhang T, Wan CY, Mei XL, Jia P and Wang MJ: Long Non-coding RNA HULC promotes progression of bone neoplasms. Med Sci Monit 24: 5754-5760, 2018.

20. Li P, Huang R, Huang T, Cheng S, Chen Y and Wang Z: Long non-coding RNA NEAT1 promotes proliferation, migration and invasion of human osteosarcoma cells. Int J Med Sci 15: $1227-1234,2018$

21. Gu W, Zhang E, Song L, Tu L, Wang Z, Tian F, Aikenmu K, Chu $\mathrm{G}$ and Zhao J: Long noncoding RNA HOXD-AS1 aggravates osteosarcoma carcinogenesis through epigenetically inhibiting p57 via EZH2. Biomed Pharmacother 106: 890-895, 2018.
22. Yang L, Ge D, Chen X, Qiu J, Yin Z, Zheng S and Jiang C: FOXP4-AS1 participates in the development and progression of osteosarcoma by downregulating LATS1 via binding to LSD1 and EZH2. Biochem Biophys Res Commun 502: 493-500, 2018.

23. Yu G, Liu G, Yuan D, Dai J, Cui Y and Tang X: Long non-coding RNA ANRIL is associated with a poor prognosis of osteosarcoma and promotes tumorigenesis via PI3K/Akt pathway. J Bone Oncol 11: 51-55, 2018

24. Ye H, Lin J, Yao X, Li Y, Lin X and Lu H: Overexpression of long Non-coding RNA NNT-AS1 correlates with tumor progression and poor prognosis in osteosarcoma. Cell Physiol Biochem 45: 1904-1914, 2018

25. Zhang X, Zhang Y, Mao Y and Ma X: The LncRNA PCAT1 is correlated with poor prognosis and promotes cell proliferation, invasion, migration and EMT in osteosarcoma. Onco Targets Ther 11: 629-638, 2018.

26. Li Z, Tang Y, Xing W, Dong W and Wang Z: LncRNA, CRNDE promotes osteosarcoma cell proliferation, invasion and migration by regulating Notch1 signaling and epithelial-mesenchymal transition. Exp Mol Pathol 104: 19-25, 2018.

27. Bayoumi AS, Sayed A, Broskova Z, Teoh JP, Wilson J, Su H, Tang YL and Kim IM: Crosstalk between long noncoding RNAs and MicroRNAs in health and disease. Int J Mol Sci 17: 356, 2016

28. Jiang N, Wang X, Xie X, Liao Y, Liu N, Liu J, Miao N, Shen J and Peng T: LncRNA DANCR promotes tumor progression and cancer stemness features in osteosarcoma by upregulating AXL via miR-33a-5p inhibition. Cancer Lett 405: 46-55, 2017.

29. Wang Y, Zeng X, Wang N, Zhao W, Zhang X, Teng S, Zhang Y and Lu Z: Long noncoding RNA DANCR, working as a competitive endogenous RNA, promotes ROCK1-mediated proliferation and metastasis via decoying of miR-335-5p and miR-1972 in osteosarcoma. Mol Cancer 17: 89, 2018.

30. Ma H, Su R, Feng H, Guo Y and Su G: Long noncoding RNA UCA1 promotes osteosarcoma metastasis through CREB1-mediated epithelial-mesenchymal transition and activating PI3K/AKT/mTOR pathway. J Bone Oncol 16: 100228, 2019.

31. Liao S, Xing S and Ma Y: LncRNA SNHG16 sponges miR-98-5p to regulate cellular processes in osteosarcoma. Cancer Chemother Pharmacol 83: 1065-1074, 2019.

32. Zhang N, Meng X, Mei L, Zhao C and Chen W: LncRNA DLX6-AS1 promotes tumor proliferation and metastasis in osteosarcoma through modulating miR-641/HOXA9 signaling pathway. J Cell Biochem: Mar 6, 2019 doi: 10.1002/jcb.28426 (Epub Ahead of Print)

33. Sun Z, Zhang T and Chen B: Long Non-coding RNA metastasis-associated lung adenocarcinoma transcript 1 (MALAT1) promotes proliferation and metastasis of osteosarcoma cells by targeting c-Met and SOX4 via miR-34a/c-5p and miR-449a/b. Med Sci Monit 25: 1410-1422, 2019.

34. Deng R, Zhang J and Chen J: LncRNA SNHG1 negatively regulates miRNA-101-3p to enhance the expression of ROCK1 and promote cell proliferation, migration and invasion in osteosarcoma. Int J Mol Med 43: 1157-1166, 2019.

35. Wang J, Cao L, Wu J and Wang Q: Long non-coding RNA SNHG1 regulates NOB1 expression by sponging miR-326 and promotes tumorigenesis in osteosarcoma. Int J Oncol 52: 77-88, 2018.

36. Dai J, Xu L, Hu X, Han G, Jiang H, Sun H, Zhu G and Tang X: Long noncoding RNA OIP5-AS1 accelerates CDK14 expression to promote osteosarcoma tumorigenesis via targeting miR-223. Biomed Pharmacother 106: 1441-1447, 2018.

37. Li Y, Zhang T, Zhang Y, Zhao X and Wang W: Targeting the FOXM1-regulated long noncoding RNA TUG1 in osteosarcoma. Cancer Sci 109: 3093-3104, 2018.

38. Li G, Liu K and Du X: Long Non-coding RNA TUG1 promotes proliferation and inhibits apoptosis of osteosarcoma cells by sponging miR-132-3p and Upregulating SOX4 expression. Yonsei Med J 59: 226-235, 2018.

39. Wang Y, Zhang R, Cheng G, Xu R and Han X: Long non-coding RNA HOXA-AS2 promotes migration and invasion by acting as a ceRNA of miR-520c-3p in osteosarcoma cells. Cell Cycle 17: 1637-1648, 2018.

40. Deng Y, Zhao F, Zhang Z, Sun F and Wang M: Long noncoding RNA SNHG7 promotes the tumor growth and epithelial-to-mesenchymal transition via regulation of miR-34a Signals in osteosarcoma. Cancer Biother Radiopharm 33: 365-372, 2018.

41. Gu Z, Hou Z, Zheng L, Wang X, Wu L and Zhang C: Long noncoding RNA LINC00858 promotes osteosarcoma through regulating miR-139-CDK14 axis. Biochem Biophys Res Commun 503: 1134-1140, 2018. 
42. Li R, Liu S, Li Y, Tang Q, Xie Y and Zhai R: Long noncoding RNA AFAP1-AS1 enhances cell proliferation and invasion in osteosarcoma through regulating miR-4695-5p/TCF4- $\beta$-catenin signaling. Mol Med Rep 18: 1616-1622, 2018.

43. Xia B, Wang L, Feng L, Tian B, Tan Y and Du B: Knockdown of long noncoding RNA CAT104 inhibits the proliferation, migration, and invasion of human osteosarcoma cells by regulating MicroRNA-381. Oncol Res 27: 89-98, 2018.

44. Yang $\mathrm{C}, \mathrm{Wu} \mathrm{K}$, Wang $\mathrm{S}$ and Wei G: Long non-coding RNA XIST promotes osteosarcoma progression by targeting YAP via miR-195-5p. J Cell Biochem 119: 5646-5656, 2018.

45. Zhao J, Zhang C, Gao Z, Wu H, Gu R and Jiang R: Long non-coding RNA ASBEL promotes osteosarcoma cell proliferation, migration, and invasion by regulating microRNA-21. J Cell Biochem 119: 6461-6469, 2018.

46. Zhou S, Yu L, Xiong M and Dai G: LncRNA SNHG12 promotes tumorigenesis and metastasis in osteosarcoma by upregulating Notch 2 by sponging miR-195-5p. Biochem Biophys Res Commun 495: 1822-1832, 2018.

47. Zhu KP, Ma XL and Zhang CL: LncRNA ODRUL contributes to osteosarcoma progression through the miR-3182/MMP2 Axis. Mol Ther 25: 2383-2393, 2017.

48. Wang W, Luo P, Guo W, Shi Y, Xu D, Zheng H and Jia L: LncRNA SNHG20 knockdown suppresses the osteosarcoma tumorigenesis through the mitochondrial apoptosis pathway by miR-139/RUNX2 axis. Biochem Biophys Res Commun 503: 1927-1933, 2018

49. Zhao D, Wang S, Chu X and Han D: LncRNA HIF2PUT inhibited osteosarcoma stem cells proliferation, migration and invasion by regulating HIF2 expression. Artif Cells Nanomed Biotechnol 47: 1342-1348, 2019.

50. Zhao W, Zhang D, Qin P, Zhang J, Cui X, Gao J, Wang J and Li J: Long non-coding RNA EPIC1 inhibits viability and invasion of osteosarcoma cells by promoting MEF2D ubiquitylation. Int J Biol Macromol 128: 566-573, 2019.

51. Chen ZX, Li QC, Liu ZZ, Liang RD and Huang B: Long non-coding RNA RAB11B-AS1 prevents osteosarcoma proliferation via its sense gene RAB11B. Zhonghua Yi Xue Za Zhi 98 2509-2514, 2018 (In Chinese).

52. Qu G, Ma Z, Tong W and Yang J: LncRNA WWOX-AS1 inhibits the proliferation, migration and invasion of osteosarcoma cells. Mol Med Rep 18: 779-788, 2018.

53. Zhao J and Ma ST: Downregulation of LncRNA H19 inhibits migration and invasion of human osteosarcoma through the NF-кB pathway. Mol Med Rep 17: 7388-7394, 2018.

54. Zhang ZC, Tang C, Dong Y, Zhang J, Yuan T, Tao SC and Li XL: Targeting the long noncoding RNA MALAT1 blocks the pro-angiogenic effects of osteosarcoma and suppresses tumour growth. Int J Biol Sci 13: 1398-1408, 2017.

55. Zhang SZ, Cai L and Li B: MEG3 long non-coding RNA prevents cell growth and metastasis of osteosarcoma. Bratisl Lek Listy 118: 632-636, 2017.

56. Sun L, Yang C, Xu J, Feng Y, Wang L and Cui T: Long noncoding RNA EWSAT1 promotes osteosarcoma cell growth and metastasis through suppression of MEG3 expression. DNA Cell Biol 35: 812-818, 2016.

57. Guo W, Jiang H, Li H, Li F, Yu Q, Liu Y, Jiang W and Zhang M: LncRNA-SRA1 suppresses osteosarcoma cell proliferation while promoting cell apoptosis. Technol Cancer Res Treat 18: $1533033819841438,2019$.

58. Fei D, Zhang X, Liu J, Tan L, Xing J, Zhao D and Zhang Y: Long noncoding RNA FER1L4 suppresses tumorigenesis by regulating the expression of PTEN targeting miR-18a-5p in osteosarcoma. Cell Physiol Biochem 51: 1364-1375, 2018.
59. Han W and Liu J: LncRNA-p21 inhibited the proliferation of osteosarcoma cells via the miR-130b/PTEN/AKT signaling pathway. Biomed Pharmacother 97: 911-918, 2018.

60. Yang C, Wang G, Yang J and Wang L: Long noncoding RNA NBAT1 negatively modulates growth and metastasis of osteosarcoma cells through suppression of miR-21. Am J Cancer Res 7: 2009-2019, 2017.

61. Sun ZY, Jian YK, Zhu HY and Li B: LncRNAPVT1 targets miR-152 to enhance chemoresistance of osteosarcoma to gemcitabine through activating c-MET/PI3K/AKT pathway. Pathol Res Pract 215: 555-563, 2019.

62. Qi X, Yu XJ, Wang XM, Song TN, Zhang J, Guo XZ, Li GJ and Shao M: Knockdown of KCNQ1OT1 suppresses cell invasion and sensitizes osteosarcoma cells to CDDP by upregulating DNMT1-mediated Kcnq1 expression. Mol Ther Nucleic Acids 17: 804-818, 2019.

63. Zhou Q, Hu T and Xu Y: Anticancer potential of TUG1 knockdown in cisplatin-resistant osteosarcoma through inhibition of MET/Akt signalling. J Drug Target 28: 204-211, 2020.

64. Song L, Zhou Z, Gan Y, Li P, Xu Y, Zhang Z, Luo F, Xu J, Zhou Q and Dai F: Long noncoding RNA OIP5-AS1 causes cisplatin resistance in osteosarcoma through inducing the LPAAT $\beta / \mathrm{PI} 3 \mathrm{~K} / \mathrm{AKT} / \mathrm{mTOR}$ signaling pathway by sponging the miR-340-5p. J Cell Biochem 120: 9656-9666, 2019.

65. Li G and Zhu Y: Effect of LncRNA ANRIL knockdown on proliferation and cisplatin chemoresistance of osteosarcoma cells in vitro. Pathol Res Pract 215: 931-938, 2019.

66. Zhou B, Li L, Li Y, Sun H and Zeng C: Long noncoding RNA SNHG12 mediates doxorubicin resistance of osteosarcoma via miR-320a/MCL1 axis. Biomed Pharmacother 106: 850-857, 2018.

67. Zhang CL, Zhu KP and Ma XL: Antisense LncRNA FOXC2-AS1 promotes doxorubicin resistance in osteosarcoma by increasing the expression of FOXC2. Cancer Lett 396: 66-75, 2017.

68. Han Z and Shi L: Long non-coding RNA LUCAT1 modulates methotrexate resistance in osteosarcoma via miR-200c/ABCB1 axis. Biochem Biophys Res Commun 495: 947-953, 2018.

69. Li Z, Zhao L and Wang Q: Overexpression of long non-coding RNA HOTTIP increases chemoresistance of osteosarcoma cell by activating the Wnt/ $\beta$-catenin pathway. Am J Transl Res 8: 2385-2393, 2016

70. Hu T, Fei Z, Su H, Xie R and Chen L: Polydatin inhibits proliferation and promotes apoptosis of doxorubicin-resistant osteosarcoma through LncRNA TUG1 mediated suppression of Akt signaling. Toxicol Appl Pharmacol 371: 55-62, 2019.

71. Yarmishyn AA and Kurochkin IV: Long noncoding RNAs: A potential novel class of cancer biomarkers. Front Genet 6: 145, 2015.

72. Shi T, Gao G and Cao Y: Long noncoding RNAs as novel biomarkers have a promising future in cancer diagnostics. Dis Markers 2016: 9085195, 2016.

73. Begolli R, Sideris N and Giakountis A: LncRNAs as chromatin regulators in cancer: From molecular function to clinical potential. Cancers (Basel) 11: 1524, 2019.

74. Miranda-Castro R, de-Los-Santos-Álvarez $N$ and Lobo-Castañón MJ: Long noncoding RNAs: From genomic junk to rising stars in the early detection of cancer. Anal Bioanal Chem 411: 4265-4275, 2019.

This work is licensed under a Creative Commons Attribution-NonCommercial-NoDerivatives 4.0 International (CC BY-NC-ND 4.0) License. 